\title{
The study of 6-DOF motion simulation platform for passenger
}

\author{
ship \\ Zhang rukai ${ }^{1, a,{ }^{*}}$ \\ ${ }^{1}$ Tianjin maritime college, Tianjin 300350, china \\ akaidesky@163.com
}

\begin{abstract}
The Stewart platform is adopted for passage ship simulator. And 6-DOF ship motion model is used for the motion control of the Stewart platform. Responding model was use as ship motion model, and assuming that the degrees of freedom of ship is independent of each other. For the rolling of ship is harder than other motion, in order to improve the effect of the simulation, separated modeling of rolling of the ship is adopted. The nonlinear rolling of the ship is researched, and the multiscale method is adopted to analysis differential equation of nonlinear rolling motion of ships in waves.
\end{abstract}

Key Words: Stewart platform, 6-DOF ship motion, nonlinear rolling, multiscale method

\section{Introduction}

Because the popular navigation simulators are all designed for merchant ships, the motion of which is usually very slow. Due to the limitation of simulator research level, it is difficult to simulate the hard motion of ship rolling. So the stationary physical platform of existing simulator can satisfy the requirement. The immersion of the simulator can be got by the visual movement. And passenger liner, for more sensitive to the wind and wave, move harder than merchant ships. So the rolling has a large range and a long period. If the traditional static platform is adopted, operator can easily realize the passive movement and the unreality of the simulator. The six degrees of freedom movement simulation platform of navigation simulator can turns the passive movement to actively movement, and enhancing the physical reality and environment reality of the simulator.

\section{Platform choice}

The Stewart platform is adopted. The classical Stewart platform is usually made up of base-platform, payload-platform, six extension-type legs and connection hinges. And the bridge can be built on the payload-platform. The 6-DOF motion of the bridge can be realized by the six extension-type legs, under the control of the control system.

Distributed architecture will be used as its control system. And the monitoring, data processing, motion control will be performed by specialized modules respectively. On one hand the control system can complete the calculation of motion model, and send 
the control commands to the hydraulic control system. On the other hand the control system can also realize demonstrate the three-dimensional animation of the movement process of the simulation platform.

\section{Motion model establishing}

The "separation" type mathematical model, putting forward by the Japanese MMG maneuvering mathematical model group, will be adopted for building the six degrees freedom movement mathematical model of the navigation manoeuvre simulator, namely the establishment of the model will be on the base of the separated hydrodynamic motion model of the hull, propeller and rudder, and the hydrodynamic interference between the ship, paddle, rudder and environmental will be considered at the same time. By manipulating ship control equipment, consistent with the actual hardware operating equipment (telegraph, rudder, tug, cables, etc.) on appearance and function of the real ship, and setting up the environment information (wind, wave and flow), the control signal will transmit to the ship motion mathematical model. And by solving the differential equation, the real-time response of ship six degrees of freedom movement will be obtained, and Marine simulator-human interaction will be realized in the loop.

\subsection{Linear model}

Responding model will be use as ship motion model, and assuming that the degrees of freedom of ship is independent of each other. For the rolling of ship is harder than other motion, in order to improve the effect of the simulation, separated modeling of rolling of the ship is adopted. The motion on the other degrees of freedom of movement, in view of the motion amplitude is small, will be just linear approximation.

The roll motion of the ship affected by crosswind and wave can be expressed as the equation:

$$
\left(J_{x x}+J_{\text {add }}\right) \cdot \ddot{\varnothing}+D(\dot{\varnothing})+\Delta \cdot \overline{G Z}(\varnothing)=M_{\text {wind,tot }}(\emptyset, t)+M_{\text {waves }}(t)
$$

$J_{x x}$ : ship moment of inertia in air, $J_{a d d}$ : added moment of inertia, $D(\dot{\varnothing})$ : general damping moment, $\Delta$ : ship displacement, $\overline{G Z}(\varnothing)$ : restoring lever, $M_{\text {wind,tot }}(\varnothing, t)$ : total instantaneous moment due to wind, $M_{\text {waves }}(t)$ : total instantaneous moment due to waves.

For the sake of calculation convenience, the effects of wind is divided into two parts, the mean wind and the instaneous wind, when the ship is exposure in the wind, the constant wind can only reduce the ability of ship against external gust effect; while the gust is the mainly cause to make the ship capsize and anchor.

The heeling moment $\overline{\mathrm{M}}_{\text {wind }}$ due to the wind can be expressed in the formula:

$$
\bar{M}_{\text {wind }}=\frac{\rho_{\text {air }} \cdot \bar{V}_{w}{ }^{2} A_{L} C_{y}\left(H_{w}+H_{y d}\right)}{2}
$$

Where, $\rho_{\text {air }}$ : air density, $\overline{\mathrm{V}}_{\mathrm{w}}$ : mean wind speed, Hw: distance from the waterline of the center of aerodynamic pressures, Hyd: distance from the waterline of the center of hydrodynamic pressures, Cy: drag coefficient, AL: lateral projected area. 
For the influence of mean wind, we can approximate changes the ship's GZ curves as follow. First of all, the mean wind made the stable position of the ship shifts from $\Phi 0$ to $\Phi$ S. In consideration of the changes of stable position, the GZ value at the windward side becomes and the stability vanish angle becomes larger, while in the lee side shows the GZ value and stability vanish angle becomes smaller. The capsizing generally occurs at the lee side, so here only the lee side considered.

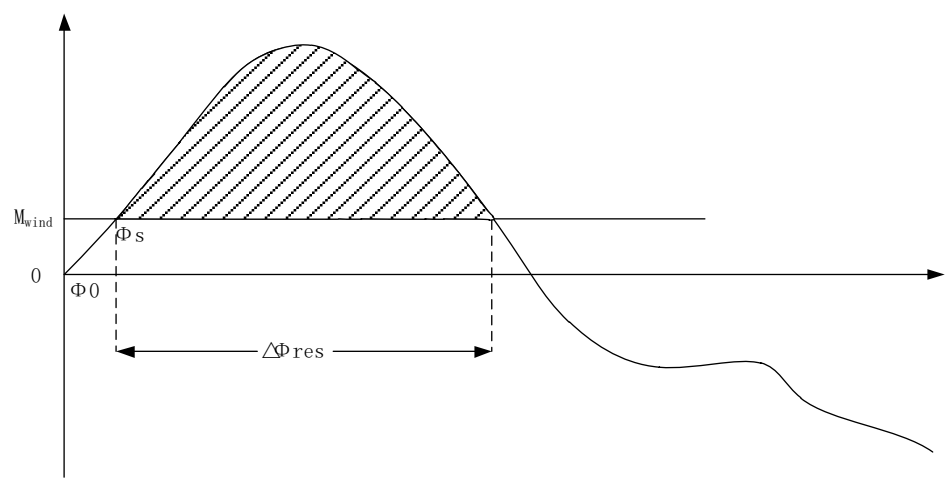

Fig.1 GZ curve schematic diagram and relevant parameters

\subsection{Nonlinear model}

On the base of linear analysis, considering nonlinear factors introduces above, the nonlinear equation can be got:

$$
\left(J_{\varphi \varphi}+\Delta J_{\varphi \varphi}\right) \ddot{\varphi}+D_{1} \dot{\varphi}+D_{3} \dot{\varphi}^{3}+D \cdot \overline{G M} \varphi+C_{3} \varphi^{3}+C_{5} \varphi^{5}=D \cdot \overline{G M} \alpha_{e o} \sin \Omega t
$$

And the two sides can be divided by $J_{\varphi \varphi}+\Delta J_{\varphi \varphi}$, then

In the equation,

$$
\ddot{\varphi}+2 \mu \dot{\varphi}+\mu_{3} \dot{\varphi}^{3}+\omega_{0}^{2} \varphi+\alpha_{3} \varphi^{3}+\alpha_{5} \varphi^{5}=f \cos (\Omega t)
$$

$$
\begin{gathered}
\mu=\frac{D_{1}}{2\left(J_{\varphi \varphi}+\Delta J_{\varphi \varphi}\right)} \\
\mu_{3}=\frac{D_{3}}{J_{\varphi \varphi}+\Delta J_{\varphi \varphi}} \\
\omega_{0}=\sqrt{\frac{D \cdot \overline{G M}}{J_{\varphi \varphi}+\Delta J_{\varphi \varphi}}} \\
\alpha_{3}=\frac{C_{3}}{J_{\varphi \varphi}+\Delta J_{\varphi \varphi}} \\
\alpha_{5}=\frac{C_{5}}{J_{\varphi \varphi}+\Delta J_{\varphi \varphi}} \\
f=\frac{J \alpha_{e o} \Omega^{2}}{J_{\varphi \varphi}+\Delta J_{\varphi \varphi}}
\end{gathered}
$$

For the sake of research convenience, the wave is assumed to propagate along a fixed direction only, and the wave crest line is infinitely long and parallel to each other.

Due to the equation of ship in waves of big rolling motion is a nonlinear equation, in order to grasp the law of nonlinear rolling motion of ship in waves, the differential equation of nonlinear rolling motion need to be solved. The multiscale method is a 
kind of approximate calculation method of high accuracy of the nonlinear problem, and will be adopted to analysis differential equation of nonlinear rolling motion of ships in waves.

In fact, subharmonic resonance is the main reason for hard rolling. In order to deal with problem, tuning factor $\sigma$ is leaded in.

And $\sigma$ :

$$
\left(\frac{1}{3} \Omega\right)^{2}=\omega_{0}^{2}+\varepsilon \sigma
$$

And equation (5) can be deformed to:

$$
\ddot{\varphi}+\frac{1}{9} \Omega^{2} \varphi+\varepsilon\left(2 \mu \dot{\varphi}+\mu_{3} \dot{\varphi}^{3}+\alpha_{3} \varphi^{3}+\alpha_{5} \varphi^{5}-\sigma \varphi\right)=f \cos (\Omega t)
$$

The first-order approximate solution of equation (6) can be got use multiscale method.

$$
\varphi(t, \varepsilon)=\varphi_{0}\left(T_{0}, T_{1}\right)+\varepsilon \varphi_{1}\left(T_{0}, T_{1}\right)
$$

According to multiscale, new independent variable is introduced to the equation

$$
T_{0}=t, T_{1}=\varepsilon t, T_{2}=\varepsilon^{2} t, \cdots, T_{n}=\varepsilon^{n} t
$$

The result can be got form the relation form, the derivative of time variable $t$ can be expressed as partial derivative of $T_{n}$.

And:

$$
\begin{gathered}
\frac{d}{d t}=\frac{\partial}{\partial T_{0}}+\varepsilon \frac{\partial}{\partial T_{1}}+\varepsilon^{2} \frac{\partial}{\partial T_{2}}+\cdots=D_{0}+\varepsilon D_{1}+\varepsilon^{2} D_{2}+\cdots \\
\frac{d^{2}}{d t^{2}}=D_{0}^{2}+2 \varepsilon D_{0} D_{1}+\varepsilon^{2}\left(D_{1}^{2}+2 D_{0} D_{2}\right)+\varepsilon^{3}\left(2 D_{0} D_{3}+2 D_{1} D_{2}\right)+\cdots
\end{gathered}
$$

In the form, $D_{0}, D_{1}, D_{2}, \cdots$ are the sign of operation of getting partial derivative of $T_{0}, T_{1}, T_{2}, \cdots$

After bring equation (9) and (10) equation to equation (6) and expanded, let the same order power factor of $\varepsilon$ same to the above equation, each order approximation equation can be got.

$$
\begin{gathered}
D_{0}{ }^{2} \varphi_{0}+\frac{1}{9} \Omega^{2} \varphi_{0}=f \cos \left(\Omega T_{0}\right) \\
D_{0}{ }^{2} \varphi_{1}+\frac{1}{9} \Omega^{2} \varphi_{1}=-2 D_{0} D_{1} \varphi_{0}-2 \mu D_{0} \varphi_{0}+\sigma \varphi_{0}-\mu_{3\left(D_{0} \varphi_{0}\right)^{3}}-\alpha_{3} \varphi_{0}{ }^{3}-\alpha_{5} \varphi_{0}{ }^{5}
\end{gathered}
$$

The solution of the zero order approximate equation, can be expressed as follow:

$$
\varphi_{0}\left(T_{0}, \quad T_{1}, T_{2}\right)=\mathrm{A}\left(T_{1}, T_{2}\right) e^{\frac{1}{3} i \Omega T_{0}}+\wedge e^{i \Omega T_{0}}+\mathrm{cc}
$$

In the form, $A$ is the plural form of the free vibration amplitude, and the amplitude of the forced vibration $\wedge$ is real number.

$$
\wedge=\frac{f}{2\left(\varphi_{0}{ }^{2}-\Omega^{2}\right)}
$$

\section{Conclusion}

In order to improve the simulation degree, the Stewart platform is adopted for passage ship simulator. And 6-DOF ship motion model is used for the motion control of the Stewart platform. For the rolling of ship is harder than other motion, in order to 
improve the effect of the simulation, the nonlinear rolling of the ship is researched, and the multiscale method is adopted to analysis differential equation of nonlinear rolling motion of ships in waves. So the immersion will be more stronger than the passive movement。

\section{Acknowledgments}

This work was financially supported by the Tianjin Maritime College technique development project "The design of practical teaching demonstration system of BRM course suitable for operational class crew”(Project No.:201610), the People's Republic of China。

\section{Reference}

[1] Li Weijia, Gao Shilun, Research of Six—Degree-of—Freedom Motion Base for Submarine Simulator for Research and Development. Huazhong University of Science and Technology, 2001.

[2] Xu Peng, Dynamic Modeling and Simulation of the Stewart Platform as one of 6-DOF Parallel Mechanism, Chongqing University, 2005.03

[3] Zhang xiufeng, The study of 6-DOF ship motion mathematical model in navigation simulator, Dalian maritime university,2009.

[4] Scamardella A, Piscopo V. Passenger ship seakeeping optimization by the Overall Motion Sickness Incidence[J]. Ocean Engineering, 2014, 76(1):86-97.

[5] Tamura Y, Arima M. Measurement And Analysis of Ship Motion of a High-speed Passenger Craft[J]. 2006. 